\title{
Dual wavelength continuous wave laser using a birefringent filter
}

\author{
C. G. Treviño-Palacios \\ carlost@inaoep.mx
}

\section{O. J. Zapata-Nava}

\section{E. V. Mejía-Uriarte}

\section{N. Qureshi \\ G. Paz-Martínez \\ O. Kolokolstev}

\begin{abstract}
Instituto Nacional de Astrofísica, Óptica y Electrónica, Luis Enrique Erro 1 Sta Ma Tonanztintla Puebla 72840 Pue México

Centro de Ciencias Aplicadas y Desarrollo Tecnológico, Universidad Nacional Autónoma de México, Ciudad Universitaria, 04510, México

Instituto Nacional de Astrofísica, Óptica y Electrónica, Luis Enrique Erro 1 Sta Ma Tonanztintla Puebla 72840 Pue México

Instituto de Física, Benemérita Universidad Autónoma de Puebla, Apdo Postal J-48, Puebla 72570, Pue México

Centro de Ciencias Aplicadas y Desarrollo Tecnológico, Universidad Nacional Autónoma de México, Ciudad Universitaria, 04510, México

Centro de Ciencias Aplicadas y Desarrollo Tecnológico, Universidad Nacional Autónoma de México, Ciudad Universitaria, 04510, México

Centro de Ciencias Aplicadas y Desarrollo Tecnológico, Universidad Nacional Autónoma de México, Ciudad Universitaria, 04510, México

Centro de Ciencias Aplicadas y Desarrollo Tecnológico, Universidad Nacional Autónoma de México, Ciudad Universitaria, 04510, México
\end{abstract}

We report simultaneous dual wavelength continuous laser emission with minimum cavity elements. Tunable dual wavelength emission between $805 \mathrm{~nm}$ and $840 \mathrm{~nm}$ was observed with controlled peak separation around two nanometers, which corresponds to approximately one terahertz. Dual wavelength laser operation is possible using a novel intracavity two plate birefringent filtering element. [DOI: http://dx.doi.org/10.2971/jeos.2013.13021]

Keywords: Birefringent filter, wavelength filtering, cavity design

\section{INTRODUCTION}

Laser source emission is determined by the gain medium and resonator characteristics in which single or multiple wavelengths have been obtained. Multiple wavelengths can produce wavelength beating and extends the capabilities of a laser source by multiple wavelength engineered emission in which the minimum independent obtainable wavelengths are two. Two independent engineered wavelengths, either laser has been used in optical coherence tomography (OCT) $[1,2]$, optical shop testing [3], commercial fiber communication systems [4], atom interferometry [5], spectroscopy and even to detect parasites in water [7]. Double wavelength emission has been obtained using diode lasers, fiber lasers and dye lasers [9]-[14]. Sources with emission in two wavelengths using titanium sapphire lasers have also been explored using coupled cavities, double-prism dispersion cavities, acousto-optic tunable filters, and with two independent seed injection lasers [15]-[22]. The use of a birefringent filter (BRF) element for dual wavelength (DW) pulsed operation with peak separation larger than $100 \mathrm{~nm}$ has also been reported [23].

In this paper we present a tunable continuous wave $(\mathrm{CW})$ dual wavelength configuration with minimum cavity elements realized on a Ti:Sapphire laser. The DW operation is based on a novel BRF designed for dual wavelength transmission. The peak separation observed is close to two nanometers, corresponding approximately to one terahertz in separation between the two emission peaks.

\section{BIREFRINGENT FILTER}

The combination of several birefringent plates in a filtering system was first introduced by Lyot in 1933. In his design the plates with optical axes aligned have lengths cascaded by a factor of two, with perfect entrance and exit polarizers on each element. Therefore the free spectral range (FSR) of the plates is thus repeatedly cut in half. The product of their single transfer functions can be used to determine the transmission of the overall filtering system.

As a laser tuning filter the elements of a BRF are oriented at the Brewster angle $\left(\theta_{B}\right)$. Such BRFs are used intracavity for laser frequency tuning in the form of a cascaded set of filters whose lengths have an integer length relation in the form $1: 2: \ldots: 2 n$, where the partial polarizing effect of the system reaches high efficiency due to the oscillation of the radiation in the laser cavity [24]. The thickness of the plates in a BRF, known as $\lambda$ - 


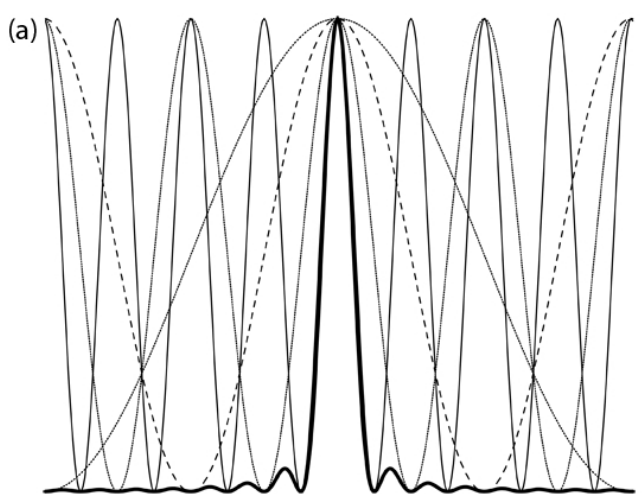

(b)

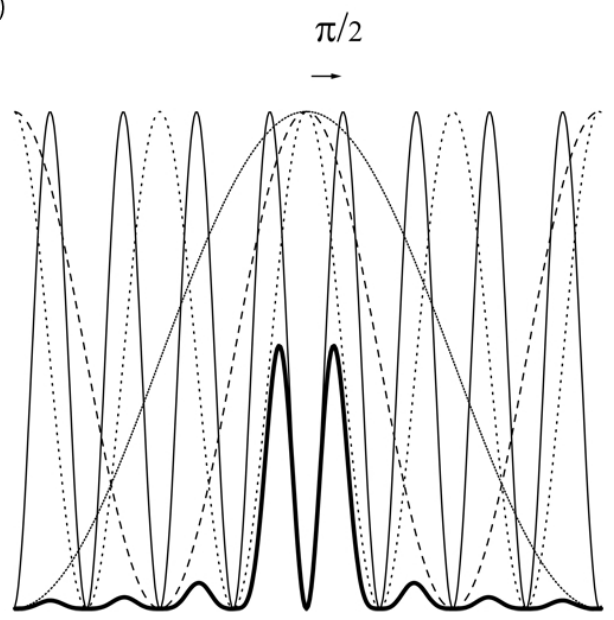

FIG. 1 Transmission of BRF plates between polarizers. The thicknesses are in ratios 1:2:4:8 with individual response in dash-dotted:dashed:dotted:solid respectively. The overall transmission (thick line) is observed when (a) the plates' optical axes are aligned and (b) the thickest plate's optical axis is rotated by $\pi / 2$ with respect to the other optical axes.

plates, is designed to allow for a retardation of multiples of $2 \pi$ at a given wavelength (Figure 1(a)).

If the thickest plate of a BRF, which has the fastest oscillating response, is rotated and/or tilted in such a way that its phaseshift is $\pi / 2$ out of phase with respect to the other plates, the transfer function of this plate has its minimum exactly at the wavelength where the other plates of the filter show a maximum. The convolution of the transfer functions then results in two peaks instead of one (Figure 1(b)). This effect can be achieved for various combinations of tilt and rotation angles by choosing different plate orders. For these different combinations the FSR also changes as a consequence of a larger or shorter optical length inside the birefringent material. Although the system departs from the unit transmission condition, the result is that the separation of the two filtered peaks can be controlled and a dual frequency filtered spectrum is obtained. As a consequence of this deviation there is a reduction in the overall transmission of the BRF and an increase in induced losses. The filter induced loss is a tolerable effect, which is diminished using large thicknesses ratios. As in a standard laser tuning BRF, the bandwidth of the transmitted peaks is determined by the number of passes through the filter which is controlled in a laser by the cavity mirrors' reflectivity. A detailed explanation of this dual wavelength filter is given elsewhere, including the body of a patent $[25,26]$.

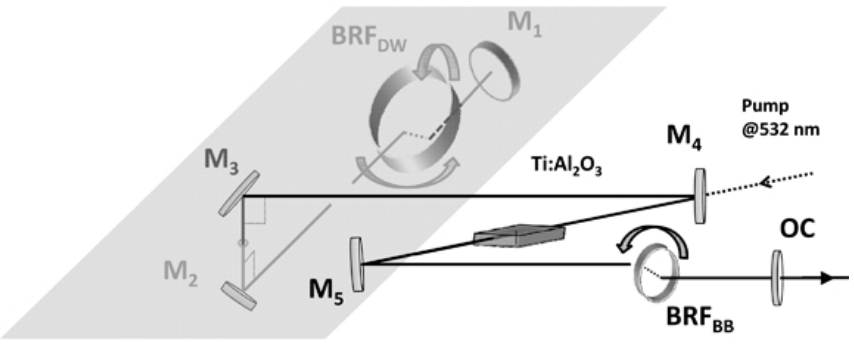

FIG. 2 Dual wavelength laser setup based on a Ti: $\mathrm{Al}_{2} \mathrm{O}_{3}$ crystal. $\mathrm{M}_{1}$ to $\mathrm{M}_{5}$ are dielectric IR mirrors, $M_{4}$ is a dichroic mirror. OC: Output Coupler. $M_{2}$ and $M_{3}$ form a $90^{\circ}$ periscope. $\mathrm{BRF}_{B B}$ is the narrow broadband filter and $\mathrm{BRF}_{D W}$ is the thick filter as described in the text.

\section{EXPERIMENTAL SETUP}

In order to realize dual wavelength emission we used a Ti:Sapphire crystal (Ti:Al2O3) which is a known inhomogeneous laser gain medium capable of dual wavelength operation [22]. We have designed a DW-BRF for operation at $810 \mathrm{~nm}$ with a $1 \mathrm{THz}$ frequency separation between the two peaks. This BRF consists of two quartz plates $\left(\mathrm{n}_{0}=1.5426\right.$, $\left.\mathrm{n}_{\mathcal{e}}=1.5517\right)$ with a $1: 16$ thickness ratio at $\theta_{B}\left(\approx 57^{\circ}\right)$. The thicknesses of the birefringent plates at normal incidence are 2.082 $\mathrm{mm}$ for the thin broadband plate $\left(\mathrm{BRF}_{B B}\right)$ and $33.315 \mathrm{~mm}$ for the thick plate $\left(\mathrm{BRF}_{D W}\right)$ to produce the double wavelength filtering. The birefringent plates were cut from the same quartz crystal and placed on a 1 inch mount for the $\mathrm{BRF}_{B B}$ plate and a 2 inch mount for the $\mathrm{BRF}_{D W}$ plate to allow for a clear aperture at $\theta_{B}$.

We used the Z-fold cavity depicted in Figure 2. The gain medium is a $7 \mathrm{~mm}$ long $\mathrm{Ti}: \mathrm{Al}_{2} \mathrm{O}_{3}$ crystal with Brewster windows from Del Mar Photonics. A mirror with linear transmission between $3 \%$ at $800 \mathrm{~nm}$ and $5 \%$ at $850 \mathrm{~nm}$ was used as output coupler (OC). The cavity was completed with dielectric mirrors: $\mathrm{M}_{1}, \mathrm{M}_{2}$ and $\mathrm{M}_{3}$ are flat mirrors, $\mathrm{M}_{4}$ and $\mathrm{M}_{5}$ are $100 \mathrm{~mm}$ curved mirrors to form a stable cavity. The cavity was pumped through the dichroic mirror $\mathrm{M}_{4}$.

The two BRF plates were placed on opposite sides of the gain medium with independent tilt and rotation. The thin broadband $\mathrm{BRF}_{B B}$ was placed vertically close to $\theta_{B}$ operating on horizontal polarization and rotated perpendicular to the path for coarse tuning of the laser. The large $\mathrm{BRF}_{D W}$ was placed horizontally, due to its weight, with fine tilt and rotation capability close to $\theta_{B}$, thus operating with vertical polarization. A $90^{\circ}$ periscope formed with $\mathrm{M}_{2}$ and $\mathrm{M}_{3}$ was used to change the polarization accordingly. The laser was pumped with a CW doubled Nd3+:YVO4 laser (Coherent Verdi V5) capable of producing up to $5.5 \mathrm{~W}$ at $532 \mathrm{~nm}$. This setup is simple to align and has minimum cavity elements. It avoids the use of multiple trajectories or external active elements as reported in other dual wavelength systems [10]-[17].

\subsection{Laser performance}

With the cavity fully aligned and only the $\mathrm{BRF}_{B B}$ plate in the cavity, the laser has tunability between $805 \mathrm{~nm}$ and 840 $\mathrm{nm}$. The spectrum was monitored using a fast monochroma- 


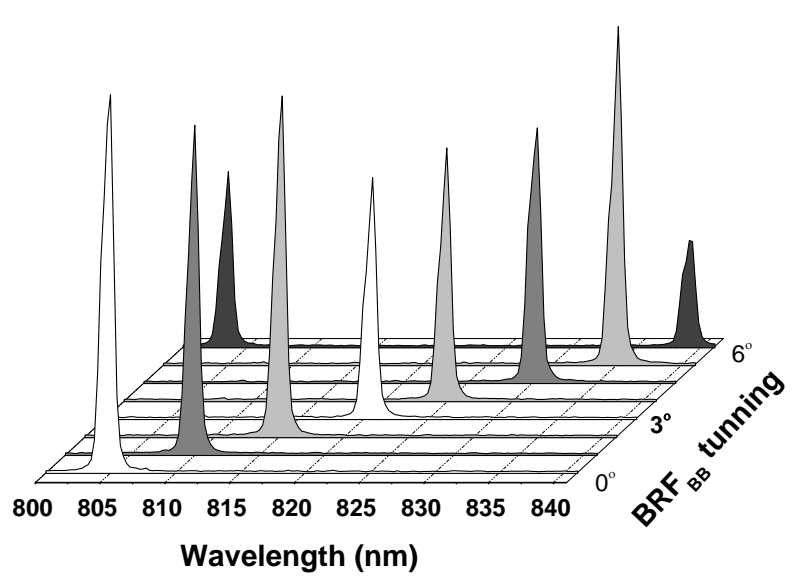

FIG. 3 Laser tuning with only the $\mathrm{BRF}_{B B}$ plate in the cavity. Notice the dual wavelength operation at the edge of the tuning range (black spectra).

tor (Ocean Optics HR4000). This tuning range corresponds to one FSR of the plate. It was obtained by rotating the plate close to $6^{\circ}$ (Figure 3 ).

With only the $\mathrm{BRF}_{B B}$ plate in the cavity the system is a standard tunable CW Ti:Sapphire laser. Except that if we play close attention to the extent of the tuning range, when the laser is close to the edge of the tuning range, on occasion there was double wavelength emission with peaks approximately $40 \mathrm{~nm}$ apart due to the balance between gain and cavity losses.

\section{DUAL WAVELENGTH OPERATION}

With both birefringent plates $\left(\mathrm{BRF}_{B B}\right.$ and $\left.\mathrm{BRF}_{D W}\right)$ placed in the cavity close to $\theta_{B}$, with high pump power we can observe DW emission with different spectral separations $(\Delta v)$ for various angle combinations of the birefringent plates. For example, in Figures 4 we observe a spectra with double wavelength taken with a high resolution monochromator (Princenton Instruments Acton SP2300 with R955 Hamamatsu Photomultiplier). Within the envelope (dotted line) there are intensity fluctuations due to gain competition and etalon effects from the $\mathrm{BRF}_{B B}$.

The conditions for DW operation were obtained by analyzing the laser in different configurations. Without the BRF, or free run configuration, the laser operates at $814.6 \mathrm{~nm}$. We obtained the laser characteristic curve (laser output as function of pump power) at this wavelength for: (A) free run; (B) with only the $\mathrm{BRF}_{B B}$ in the cavity; with both $\mathrm{BRF}_{B B}$ and $\mathrm{BRF}_{D W}$ in (C) single wavelength and (D) DW operation (Figure 5).

From these curves we obtained (Table 1) the laser pump threshold $\left(\mathrm{P}_{t h}\right)$, extraction efficiency $(\eta)$, and maximum output $\left(\mathrm{I}_{\max }\right)$ for the configurations studied. For single wavelength operation we observe a threshold increase and extraction efficiency decrease when the birefringent plates are inserted due to internal losses of the quartz plates and filter strengthening, respectively.

When the system operates in double wavelength emission the

\begin{tabular}{|c|c|c|c|}
\hline & $\mathrm{P}_{T h}(\mathrm{~W})$ & $\eta(\%)$ & $\mathrm{I}_{\max }(\mathrm{mW})$ \\
\hline Free run & $1.79 \pm 0.06$ & $3.50 \pm 0.04$ & $132 \pm 1$ \\
\hline BRF $_{B B}$ & $2.04 \pm 0.05$ & $3.47 \pm 0.04$ & $120 \pm 1$ \\
\hline $\begin{array}{c}\mathrm{BRF}_{D W}+\mathrm{BRF}_{B B} \\
(1 \lambda)\end{array}$ & $2.52 \pm 0.04$ & $2.76 \pm 0.03$ & $80 \pm 1$ \\
\hline $\begin{array}{c}\mathrm{BRF}_{D W}+\mathrm{BRF}_{B B} \\
(2 \lambda)\end{array}$ & $3.14 \pm 0.05$ & $2.59 \pm 0.05$ & $64 \pm 1$ \\
\hline
\end{tabular}

TABLE 1 Pump threshold $\left(\mathrm{P}_{T h}\right)$, extraction efficiency $(\eta)$ and maximum output $\left(\mathrm{I}_{\text {max }}\right)$ for different laser configurations.

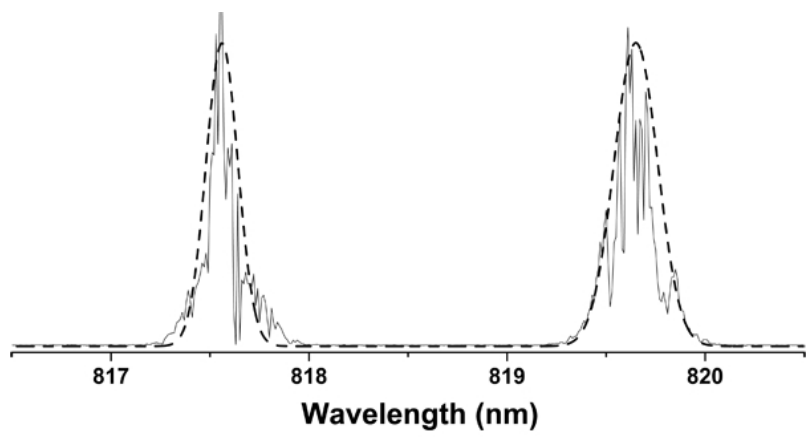

FIG. 4 Ti:Sapphire laser dual wavelength emission ( $817.57 \mathrm{~nm}$ and $819.63 \mathrm{~nm}$ ) with spectral separation $\Delta v=0.953 \mathrm{THz}$.

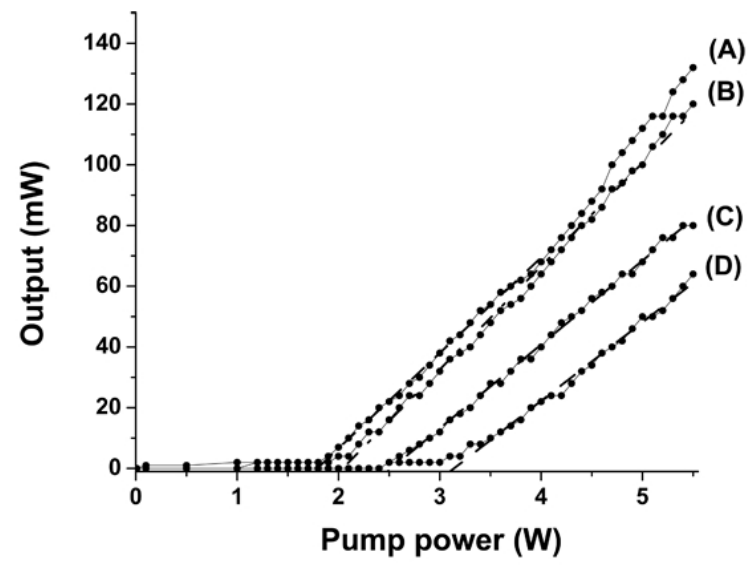

FIG. 5 Laser emission characteristic at $814.6 \mathrm{~nm}$ for (A) cavity without BRF, (B) with only $B R F B B$ in the cavity; $(C)$ with $B_{B R}$ and $B_{B R F}$ in the cavity in single wavelength operation and $(D) B R F_{B B}$ and $B R F_{D W}$ in the cavity in dual wavelength operation.

BRF no longer exhibits unit transmission and additional losses are induced from the convolution among the birefringent plates (Figure 1(b)) increasing further the laser threshold. But the inhomogeneous gain of the Ti:Sapphire is large enough to sustain DW lasing. We determined the double wavelength tuning capabilities of the system with the complete BRF in the cavity and the $\mathrm{BRF}_{D W}$ plate at fixed angle. We fine tuned the system by rotating the $\mathrm{BRF}_{B B}$ plate close to $6^{\circ}$ by an entire FSR cycle (Figure 6). We observe that the system switches between single (white spectra) and double (grey spectra) wavelength emission while turning the plate. Within a complete $\mathrm{BRF}_{B B}$ FSR cycle (dark spectra) we observe 16 positions where there is a double wavelength emission due to the spectral response of the $\mathrm{BRF}_{D W}$ plate, in accordance with the BRF design.

Double wavelength laser emission second harmonic generation (SHG) and sum frequency generation (SFG) using a $7 \mathrm{~mm}$ 


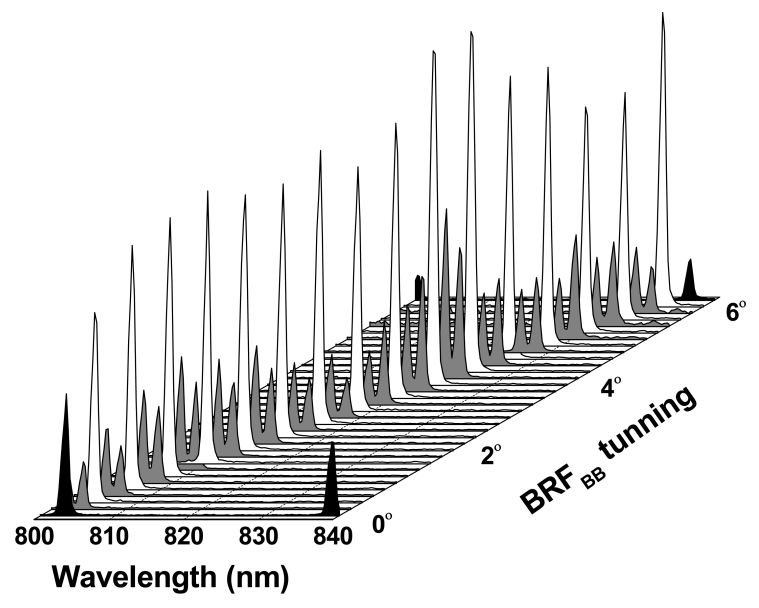

FIG. 6 Laser tuning as function of $\mathrm{BRF}_{B B}$ rotation with both $\mathrm{BRF}_{B B}$ and $\mathrm{BRF}_{D W}$ in the cavity. The laser switches between single wavelength operation (white spectra) and double wavelength operation (grey spectra) within the FSR range of the $B_{B B} F_{B B}$ (black spectra).

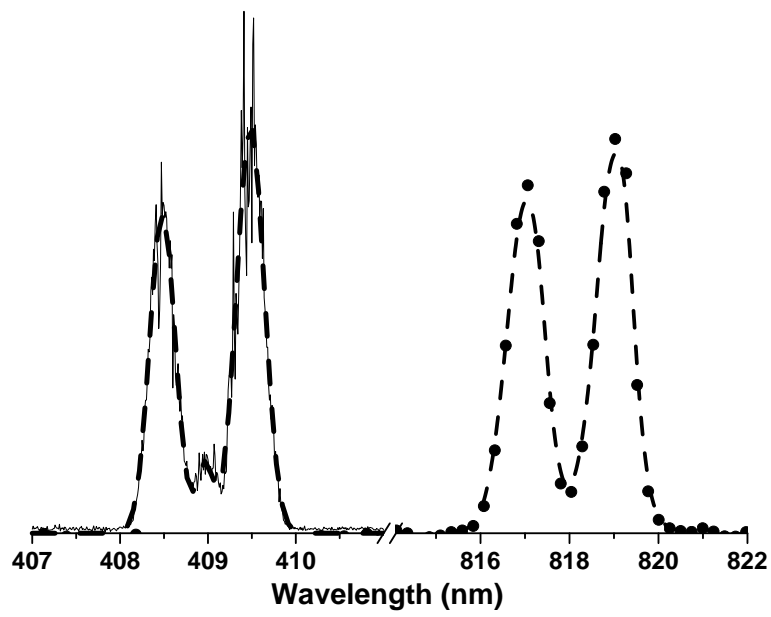

FIG. 7 (Right) Dual wavelength lasing (816.96 and $818.97 \mathrm{~nm}, \Delta v=0.901 \mathrm{THz})$ and (Left) signal through a BBO crystal producing SHG (408.48 and $409.48 \mathrm{~nm}$ ) and SFG $(408.96 \mathrm{~nm})$. The relative height of the converted peaks are: $1.00(408.48 \mathrm{~nm}), 0.28$ $(408.96 \mathrm{~nm})$, and $1.25(409.48 \mathrm{~nm})$.

$\mathrm{BBO}\left(\mathrm{BaB}_{2} \mathrm{O}_{4}\right)$ crystal was observed (Figure 7). SFG is only observed when both signals are present at the same time confirming simultaneous double wavelength operation, in spite of laser fluctuations. The double wavelength emission is unstable and will require feedback to improve the stability of the system. This laser system emitting in two wavelengths simultaneously could potentially be used to generate new wavelengths by nonlinear mixing, in particular due to the wavelength design a source in the millimeter-Terahertz wavelength range.

\section{CONCLUSIONS}

In conclusion we have demonstrated an all optical simultaneous continuous wave dual wavelength titanium sapphire laser with minimal cavity elements and easy alignment. The dual wavelength is achieved using a two-plate BRF with dual wavelength filtering with design tunable spectral separation around one terahertz.

\section{ACKNOWLEDGEMENTS}

The authors acknowledge support from grants ICyTDF PIUTE10-71 and PAPIIT IT-110811.

\section{References}

[1] Y. Mao, S. Chang, E. Murdock, and C. Flueraru, "Simultaneous dual-wavelength-band common-path swept-source optical coherence tomography with single polygon mirror scanner," Opt. Lett. 36, 1990-1992 (2011).

[2] S. Chang, Y. Mao, and C. Flueraru, "Dual-Source Swept-Source Optical Coherence Tomography Reconstructed on Integrated Spectrum," International Journal of Optics 2012, 565823 (2012).

[3] A. García-Arellano, F. Granados-Agustín, M. Campos-García, and A. Cornejo-Rodríguez, "Ronchi test with equivalent wavelength," Appl. Optics 51, 3071-3080 (2012).

[4] http://www.ozoptics.com/ALLNEW_PDF/DTS0009.pdf

[5] V. Ménoret, R. Geiger, G. Stern, N. Zahzam, B. Battelier, A. Bresson, A. Landragin, and P. Bouyer, "Dual-wavelength laser source for onboard atom interferometry," Opt. Lett. 36, 4128-4130 (2011).

[6] S. Shibata, "Dual-Wavelength Spectrophotometry," Angew. Chem. Int. Ed. Engl. 15, 673-679 (1976).

[7] S. Buaprathoom, S. Pedley, and S. J. Sweeney, "Dual wavelength multiple-angle light scattering system for cryptosporidium detection," Proc. SPIE 8427, Biophotonics: Photonic Solutions for Better Health Care III, 84272K (2012).

[8] P. Gu, F. Chang, M. Tani, K. Sakai, and C. Pan, "Ceneration of Coherent CW-Terahertz Radiation Using a Tunable Dual-Wavelength External Cavity Láser Diode," Jpn. J. Appl. Phys. 38, 1246-1248 (1999).

[9] M. Matus, M. Kolesik, J. V. Moloney, M. Hofmann, and S. W. Koch, "Dynamics of two-color laser systems with spectrally filtered feedback," J. Opt. Soc. Am. B 21, 1758-1771 (2004).

[10] R. M. Sova, C.-S. Kim, and J. U. Kang, "Tunable Dual-Wavelength All-PM Fiber Ring Laser," IEEE Phot. Tech. Lett. 14, 287-289 (2002).

[11] X. Liu, X. Yang, F. Lu, J. Ng, X. Zhou, and C. Lu, "Stable and uniform dual-wavelength erbium-doped fiber laser based on fiber Bragg gratings and photonic crystal fiber," Opt. Express 13(1), 142-147 (2005).

[12] L. Guo, R. Lan, H. Liu, H. Yu, H. Zhang, J. Wang, D. Hu, et al., "1319 $\mathrm{nm}$ and $1338 \mathrm{~nm}$ dual-wavelength operation of LD end-pumped Nd:YAG ceramic laser," Opt. Express 18(9), 9098-9106 (2010).

[13] B. Yao, Y. Tian, G. Li, and Y. Wang, "InGaAs/GaAs saturable absorber for diode-pumped passively Q-switched dual-wavelength Tm:YAP lasers," Opt. Express 18(13), 13574-13579 (2010).

[14] H. Yoshioka, S. Nakamura, T. Ogama, and S. Wada, "Dualwavelength mode-locked Yb:YAG ceramic laser in single cavity," Opt. Express 18(2), 1479-1486 (2010).

[15] H. Maestre, A. J. Torregrosa, C. R. Fernández-Pousa, M. L. Rico, and J. Capmany, "Dual-wavelength green laser with a 4.5 $\mathrm{THz}$ frequency difference based on self-frequency- doubling in $\mathrm{Nd} 3+$-doped aperiodically poled lithium niobate," Opt. Lett. 33, 1008-1010 (2008).

[16] 0. J. Zapata-Nava, C. G. Treviño-Palacios, M. D. Iturbe-Castillo, and P. Rodriguez-Montero, "Grating cavity dual wavelength dye laser," Opt. Express 19, 3483-3493 (2011). 
[17] D. Xin, P. Ming, Y. Xuan-Yi, W. Xiao-Heng, Z. Shao-Min, Z. Heng, W. Rui, et al., "An All-Solid-State Tunable Dual-Wavelength Ti:Sapphire Laser with Quasi-Continuous-Wave Outputs," Chin. Phys. Lett. 24(7), 1938-1940 (2007).

[18] Y. Fujii, and M. Katsuragawa, "Dual-frequency pulsed laser with an accurate gigahertz-beat note," Opt. Lett. 32, 3065-3067 (2007).

[19] K. Ertel, H. Linné, and J. Bösenberg, “Injection-seeded pulsed Ti:sapphire laser with novel stabilization scheme and capability of dual-wavelength operation," Appl. Optics 44, 5120-5126 (2005).

[20] K. Kawase, M. Mizuno, S. Sohma, H. Takahashi, T. Taniuchi, Y. Urata, S. Wada, et al., "Difference-frequency terahertzwave generation from 4-dimethylamino-N-methyl-4-stilbazoliumtosylate by use of an electronically tuned Ti:sapphire laser," Opt. Lett. 24, 1065-1067 (1999).

[21] M. Katsuragawa, and T. Onose, "Dual-wavelength injection-locked pulsed laser," Opt. Lett. 30, 2421-2423 (2005).
[22] N. Saito, S. Wada, and H. Tashiro, "Dual-wavelength oscillation in an electronically tuned Ti:sapphire laser," J. Opt. Soc. Am. B 18, 1288-1296 (2001).

[23] D. Xin, L. Xue, S. Quan, S. Chun-Peng, Y. Su-Jia, L. Bin, Y. XuanYi, et al., "High Power Widely Tunable Narrow Linewidth All-SolidState Pulsed Titanium-Doped Sapphire Laser," Chin. Phys. Lett. 28, 094205 (2011).

[24] M. Title, “Tunable birefringent filters," Opt. Eng. 20, 815-823 (1981).

[25] C. G. Treviño-Palacios, C. Wetzel, and 0. J. Zapata-Nava, “Design of a Dual Wavelength Birefringent Filter," in Proceedings of RIAO/OPTILAS 2007: 6th Ibero-American Conference on Optics (RIAO); 9th Latin-American Meeting on Optics, Lasers and Applications (OPTILAS), 392-397 (AIP, Sao Paulo, 2008).

[26] C. G. Treviño-Palacios, and C. Wetzel, "Dual wavelength birefringent filter," Mexican Patent MX280131B (2010). 